\title{
The Spatial and Temporal Prognosis of Oilseed Yield in Shandong Province
}

\author{
Yujian Yang ${ }^{1, *}$, Jianhua Zhu ${ }^{1}$, Shubo $\mathrm{Wan}^{2}$, and Xiaoyan Zhang ${ }^{1}$ \\ ${ }^{1} \mathrm{~S} \& \mathrm{~T}$ Information Engineering Technology Center of \\ Shandong Academy of Agricultural Science, Jinan 250100, P.R. China \\ ${ }^{2}$ Shandong Academy of Agricultural Science, Jinan 250100, P.R. China
}

\begin{abstract}
Based on the data about oilseed yield of 87 country units in Shandong province, the paper performed the Moran's I computerization to analyze the spatial autocorrelation characteristics of the oilseed yield on country level. Results showed that the spatial pattern of the oilseed yield presented the significant agglomeration characteristics, the Moran's I coefficient of 14 country units was noted quadrant $\mathrm{HH}$, which displayed the country units with a high oilseed yield (above the average) surrounded by country units with high oilseed yield (above the average), the Moran's I coefficient of 4 country units was noted quadrant LH, which showed the country units with low value surrounded by country units with high values, the Moran's I coefficient of 22 country units was noted quadrant LL, which indicated the country units with low oilseed yield surrounded by country units with low oilseed yield, the autocorrelation of the other country units was not evident. The study also carried out to predict the total oilseed yield with ARIMA $(2,1,2)$ model on basis of time series data, in order to explore the trend of the total oilseed yield in Shandong province, the average relative error between observation value and prediction value is $2.12 \%$ only using statistical oilseed yield data during 1978-2008, the better reliability. In a word, Moran's I coefficient and ARIMA $(2,1,2)$ model can fairly clarify the spatial and temporal status of oilseed yield. What's more, the study is to provide a better understanding of temporal and spatial patterns of oilseed yield in Shandong province.
\end{abstract}

Keywords: Shandong Province, Oilseed yield, ARIMA model, Spatial autocorrelation.

\section{Introduction}

Yield and quality are always the core element for the oilseed study, the improving of oilseed yield is not only helpful for expanding oilseed crops areas in the acreage planted, but also developing good quality, high-yield and high-efficiency agriculture,

\footnotetext{
* This work was supported by the National Key Technology R\&D Program (2006BAD21B04-20) and Innovation Fund Projects of Shandong Academy of Agricultural Science (SAAS) (2006YCX035, 2007YCX027).
} 
furthermore, carrying out industrial restructuring and constructing the modern agriculture industry structure. From the literature retrieval, oilseed crop was mainly studied in the mechanism of oilseed growth [1].

The spatial autocorrelation was widely involved in the fields. López-Bazo et al. (2004) [2] applied these ESDA spatial tools to explore the variable the spatial agglomeration on a large regional scale, and the interior potential development on a smaller scale about farm area unit by Kathryn (2005) [3], It is reported that Jinfeng Wang (2007) [4] studied the spatial autocorrelation of regional parameters characteristics, such as air pollution processes, population mortality, and regional poverty levels. Yuming Wu $(2005,2007)$ [5] studied the spatial clustering character of the regional economic growth about 31 province units of China. Aiqing Lv (2008) [6] launched the spatial model study of factors for grain yield. However, the few study of spatial autocorrelation analysis was involved in the past years about oilseed yield on country level in Shandong province. For the time series data, an important question in the past decade has been the trend behavior of agricultural output, attention has been primarily focused on testing between the deterministic time trend model and the unit root with drift model, the ARIMA model should be useful for the long-run behavior. The time series model has certain advantage in the prediction of the total crops yield. The researcher in the past study on timing algorithm is feasible to be used to prediction production (Guifen Chen, 2009) [7]. However, the oilseed yield prediction mode based on time series data is rare reported.

In the work, we explored the comprehensive study combining spatial autocorrelation prognosis with time series data prediction. The spatial agglomeration effects of oilseed yield on country level was clarified by the autocorrelation Moran's I coefficient and the spatial patterns of oilseed yield was analyzed to understand the regional effects for adjacent country units. At the same time, based on time series data of the total oilseed yield in Shandong province, the prognosis was implemented the ARIMA study optimizing for oilseed crop industrial structure. The study focused on the temporal and spatial changes of oilseed yield, which is helpful for improving oilseed yield, so for coordinated developing primary, secondary and tertiary industries in Shandong province.

\section{Material and Methodology}

\subsection{Review of ARIMA Model and Moran's I Coefficient}

A time series is a sequence of data consisting of continuous values, this kind of model can be divided into trend component, periodical component and noisy component and the ARIMA is denoted "Auto-Regressive Integrated Moving Average", lags of the differenced series appearing in the forecasting equation are called "auto-regressive" terms, lags of the forecast errors are called "moving average" terms. Especially, the case of equation containing $p$ lags and the model for sequence $q$ lags, the model is called an $\operatorname{ARMA}(p, q)$ model [8]. If $q=0$, the process is called a pure 
autoregressive process denoted by $A R(p)$, and if $p=0$, the process is a pure moving average process denoted by $M A(q)$.

Autoregressive integrated moving average model was shortened in $\operatorname{ARIMA}(p, d, q)$, where: $p$ is the number of autoregressive terms, $d$ is the number of non-seasonal differences, and $q$ is the number of lagged forecast errors in the prediction. The following is the structure of the model.

$$
\left\{\begin{array}{l}
\Phi(B) \nabla^{d} x_{t}=\Theta(B) \varepsilon_{t} \\
E\left(\varepsilon_{t}\right)=0, \operatorname{Var}\left(\varepsilon_{t}\right)=\sigma_{\varepsilon}^{2}, E\left(\varepsilon_{t} \varepsilon_{s}\right)=0, s \neq t \\
E x_{s} \varepsilon_{t}=0, \forall s<t
\end{array}\right.
$$

Where, $\nabla^{d}=(1-B)^{d} ; \Phi(B)=1-\phi_{1} B-\cdots-\phi_{P} B^{p}$, was regarded as the polynomial coefficients for the smooth and reversible autoregressive model. In the study, we will try to stick to "unmixed" models with either only- $A R$ or only- $M A$ terms, because including both kinds of terms in the same model sometimes leads to be over-fitting of the data and non-uniqueness of the coefficients. In $\operatorname{ARIMA}(p, d, q)$ model, $\Theta(B)=1-\theta_{1} B-\cdots-\theta_{q} B^{q}$ was taken as the smoothing polynomial coefficients for the reversible autoregressive model, so formula (1) was shorten as:

$$
\nabla^{d} x_{t}=\frac{\Theta(B)}{\Phi(B)} \varepsilon_{t}
$$

Where, $\left\{\varepsilon_{t}\right\}$ denotes the white noise series for the mean value based on data, formula (2) explained the difference and combination of the model embodied the essence of $\operatorname{ARIMA}(p, d, q)$, and the differential stationary data was obtained from the suitable orders difference, then $\operatorname{ARIMA}(p, d, q)$ was implemented and transformed into the following structure.

$$
\left\{\begin{array}{l}
x_{t}=\phi_{0}+\phi_{1} x_{t-1}+\cdots+\phi_{p} x_{t-p}+\varepsilon_{t}-\theta_{1} \varepsilon_{t-1}-\cdots-\theta_{q} \varepsilon_{t-q} \\
\phi_{p} \neq 0, \theta_{q} \neq 0 \\
E\left(\varepsilon_{t}\right)=0, \operatorname{Var}\left(\varepsilon_{t}\right)=\sigma_{\varepsilon}^{2}, E\left(\varepsilon_{t} \varepsilon_{s}\right)=0, s \neq t \\
E x_{s} \varepsilon_{t}=0, \forall s<t
\end{array}\right.
$$

If $\Phi(B) x_{t}=\Theta(B) \varepsilon_{t}, \Phi(B)=1-\phi_{1} B-\cdots-\phi_{p} B^{p}$ formular (3) is regarded as the autocorrelation coefficient of $\mathrm{p}$ polynomial orders. If $\Theta(B)=1-\theta_{1} B$ $-\cdots-\theta_{q} B^{q}$, then the model is regarded as the autocorrelation coefficient of $q$ polynomial orders $[9,10,11,12]$. 
Moran's I can be used in a wide variety of circumstances. As a global statistic, Moran's I quickly indicates not only the existence of spatial autocorrelation (positive or negative), but also the degree of spatial autocorrelation. The Moran's I coefficient is given by:

$$
I=\frac{n}{S_{0}} \times \frac{\sum_{i}^{n} \sum_{j=1}^{n} w_{i j}\left(x_{i}-\bar{x}\right)\left(x_{j}-\bar{x}\right)}{\sum_{i}^{n}\left(x_{i}-\bar{x}\right)^{2}}
$$

As discussed in the above description, $I$ represents a decomposition of the global Moran`s I, a positive $I$ indicates clustering of high or low values, a negative $I$ indicates a spatial outlier.

The matrix form for

$$
I=\frac{n}{S_{0}} \times \frac{X^{\prime} W X}{X^{\prime} X}
$$

Where: $x_{i}, x_{j}$ denotes the observation of the spatial unit based on the element $x$.

$$
\bar{x}=\frac{1}{n} \sum_{i=1}^{n} x_{i}
$$

Where, $\bar{x}$ stands for the mean value, $X^{\prime}$ stands for deviation vector about $x_{i}$ and $\bar{x}(n \times 1)$, the elements $w_{j i}$ indicated the way the region $i$ is spatially connected to the region $j$, represented the topological relation. $W$ denotes the spatial matrix about $n \times n, S_{0}$ represented the sum of all of the element about spatial weight matrix, $n$ is regarded as the spatial units. If the spatial autocorrelation of observation value does not exist, the original random status was used to validate $z$ value of the hypotheses which referred to the formula (7) illustration:

$$
z(I)=\frac{I-E(I)}{\sqrt{\operatorname{var}(I)}}
$$

Where: $I \in[-1,1], I=0$ stands for the independent variables, $I>0$ stands for the positive correlation, $I<0$ stands for the negative correlation $[13,14,15,16]$.

Spatial autocorrelation can be defined as the coincidence of value similarity with site similarity. Therefore, there is the positive autocorrelation when high or low values of a spatial randomly variable tendency to cluster, and negative autocorrelation when sites tend to be surrounded by neighbors with very dissimilar values. Moran's I coefficient was regarded as the measurement degree of spatial autocorrelation character. On the whole, its value was divided into four quadrants. Quadrant I (on the top right corner) 
presents the country units with a high oilseed yield (above the average) surrounded by country units with high oilseed yield (above the average), this quadrant is usually noted HH. Quadrant II (on the top left corner) shows the country units with low value surrounded by the neighboring country units with high oilseed yield values, the quadrant is usually noted LH. Quadrant III (on the bottom left) displays the country units with low value surrounded by country units with low values, and is noted LL. Quadrant IV (on the bottom right) shows the country units with high value surrounded by country units with low values, is noted HL $[17,18]$.

\subsection{Data Acquisition}

The analysis data came from Shandong Statistical Year Book-2008, Chinese county (city) social economic statistical yearbook-2008 in Shandong province. The oilseed yield on country level was obtained from the above collected data. The spatial data of 91 country units which had the developed agriculture mainly were selected to exclude 49 district units in Shandong province. Actually, the spatial autocorrelation characteristics based on 87 country units (not including Qingyun country, Yangxin country, Changdao country and Yutai country units lack of the statistical data) were selected to computerize, the scale of the vector map is 1:500000 and the projection transformation was finished.

In this work, we find strong evidence in favor of global spatial autocorrelation for oilseed yield on country level in Shandong province. Furthermore, in view of the total oilseed yield, the ARIMA model was established to forecast the increase of oilseed yield on the basis of time series data from 1978 to 2008, so the main objective is the spatial and temporal prediction of the oilseed yield in Shandong province in the paper.

\section{Stationary Analysis and Temporal Prediction of Oilseed Yield}

\subsection{The Stationary Series Stationary Process on Time Series Data}

As you will see in the next section, the stability condition is that the characteristic roots of the polynomial must lie outside of the unit circle. It is also shown that if data sequence is a linear stochastic difference equation, the stability condition is the necessary condition for time series data to be stationary. More generally, the PACF of a stationary model process must ultimately decay toward zero beginning at lags $\mathrm{p}$, the decay pattern depends on the coefficients of the polynomial summaries some of the properties at the ACF and PACF for various ARIMA processes. 
$\mathrm{AC}$ is the autocorrelation coefficient, and PAC is the partial autocorrelation coefficient, the last two ranks number is one $Q$ statistics variable, the other is probability rank, probability rank number represented $Q$ probability value, you can see that the distribution pattern of the oilseed yield, natural number of the first rank represented the lagged period. Non-stationary time series data was transformed into the stationary time series data by ARIMA model, we take the first-difference of "oilseed yield series data" to see whether the time series data becomes the stationary process, the autocorrelation and partial autocorrelation plot was thought as the stationary tool for time series data, according to autocorrelation and partial autocorrelation plot, as is illustrated in Figure 1, the autocorrelation coefficient of oilseed yield didn't rapidly decrease zero value, which verified the non-stationary time series.

\begin{tabular}{|c|c|c|c|c|c|c|c|c|}
\hline \multicolumn{9}{|c|}{$\begin{array}{l}\text { Date: } 11 / 27 / 09 \text { Time: } 13: 57 \\
\text { Sample: } 19782008 \\
\text { Included observations: } 31\end{array}$} \\
\hline \multicolumn{2}{|c|}{ Autocorrelation } & \multicolumn{2}{|c|}{ Partial Correlation } & & $A C$ & \multirow{2}{*}{$\begin{array}{ll}\text { PAC } \\
40.804\end{array}$} & \multirow{2}{*}{$\begin{array}{l}\text { Q-Stat } \\
22.032\end{array}$} & \multirow{2}{*}{$\begin{array}{l}\text { Prob } \\
0.000\end{array}$} \\
\hline ' & & 1 & & 1 & 0.804 & & & \\
\hline ' & & 1 & 1 & 2 & 0.685 & 0.111 & 38.608 & 0.000 \\
\hline I & $\nabla$ & 1 & 1 & 3 & 0.620 & 0.118 & 52.671 & 0.000 \\
\hline I & & 1 [ & 1 & 4 & 0.515 & -0.098 & 62.704 & 0.000 \\
\hline I & $\square$ & 17 & 1 & 5 & 0.445 & 0.031 & 70.497 & 0.000 \\
\hline i & $\square$ & 1 면 & 1 & 6 & 0.338 & -0.149 & 75.171 & 0.000 \\
\hline I & 马i & 1 & 1 & 7 & 0.267 & 0.028 & 78.221 & 0.000 \\
\hline 1 & घ & 1 & 1 & 8 & 0.255 & 0.105 & 81.108 & 0.000 \\
\hline ' & घ & 15 & 1 & 9 & 0.191 & -0.070 & 82.803 & 0.000 \\
\hline 1 & ב & 1 & 1 & 10 & 0.142 & -0.022 & 83.783 & 0.000 \\
\hline I & I & 1 1 & 1 & 11 & 0.031 & -0.253 & 83.833 & 0.000 \\
\hline
\end{tabular}

Fig. 1. The autocorrelation and partial autocorrelation plot of oilseed yield from 1978 to 2008 (left)

\begin{tabular}{|c|c|c|c|c|c|c|c|}
\hline \multicolumn{8}{|c|}{$\begin{array}{l}\text { Date: } 11 / 27 / 09 \text { Time: } 13: 49 \\
\text { Sample: } 19782008 \\
\text { Included observations: } 30\end{array}$} \\
\hline Autocorrelation & Partial Co & orrelation & & $A C$ & PAC & Q-Stat & Prob \\
\hline I & , & I & & $1-0.293$ & -0.293 & 2.8493 & 0.091 \\
\hline ，回， & 回 & , & & $2-0.151$ & -0.259 & 3.6317 & 0.163 \\
\hline 17 & 17 & I & & $\begin{array}{ll}3 & 0.078\end{array}$ & -0.064 & 3.8455 & 0.279 \\
\hline 1 든 & 10 & 1 & & $4-0.190$ & -0.261 & 5.1777 & 0.270 \\
\hline ， & 1 & 1 & & $\begin{array}{ll}5 & 0.154\end{array}$ & 0.005 & 6.0929 & 0.297 \\
\hline 15 & 1 - & 1 & & $6-0.065$ & -0.121 & 6.2621 & 0.394 \\
\hline 1 1 & 10 & $i$ & & $7-0.077$ & -0.124 & 6.5118 & 0.481 \\
\hline 1 & 1 & I & & $\begin{array}{lll}8 & 0.043\end{array}$ & -0.126 & 6.5944 & 0.581 \\
\hline i & i & i & 9 & 90.043 & 0.002 & 6.6779 & 0.671 \\
\hline 161 & i & , & 10 & 0.099 & 0.086 & 7.1451 & 0.712 \\
\hline 171 & 1 & 曰 & 11 & 10.077 & 0.200 & 7.4436 & 0.762 \\
\hline
\end{tabular}

Fig. 2. The autocorrelation and partial autocorrelation plot of oilseed yield after 1-order differential transformation (right)

So data request the stability for ARIMA model, for the stationary processes, the key points to note are the following description: (1) The ACF of ARIMA model process will begin to decay at lag q, the coefficient of the ACF will satisfy the difference equation, since the characteristic roots are inside the unit circle, the autocorrelations will decay beginning at lag q.. Moreover, the spatial pattern of the autocorrelation coefficients will mimic step by step, which is suggested by the characteristic roots. (2) The PACF of ARIMA model process will begin to decay at lag $\mathrm{p}$, the coefficients of the PACF will mimic the ACF coefficients from the model, in terms of the above description, the stationary process for oilseed yield by means of the differential data in the article, after first order differential, series data trend was eliminated and the relevant information was sufficiently extracted, the variance presented the stationary characteristics, finally autocorrelation coefficient satisfied in the confidence level, which resulted into the random series data, or was noted as white noise series, referred to Figure 2. Model is recognized in terms of the autocorrelation and partial autocorrelation's function characteristics of time series sample and selected type of model, meanwhile determining the appropriate model order on basis of differential transformation. Depending on the mean value and constant variance, the time series plot presented the random oscillation characteristics with the support of the constant value, the bounder of oscillations and no evident trend and period characteristics, and no stronger change of sample, which indicated the stationary characteristics after data 1-order differential transformation about the oilseed yield, as is illustrated in Figure 3 [9,19]. 


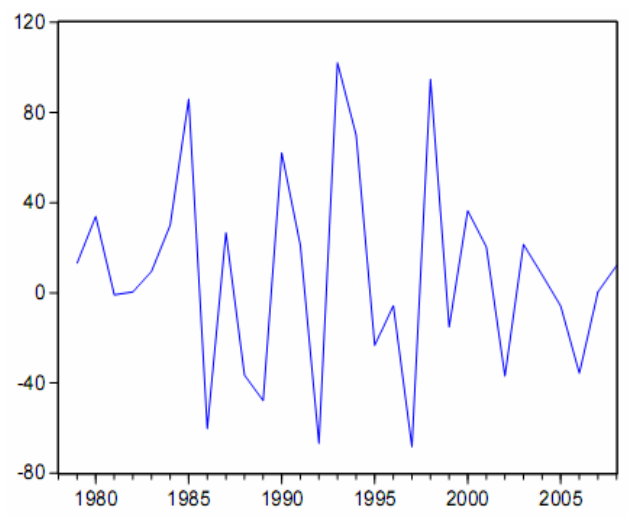

Fig. 3. Sequence plot on 1-order differential data of oilseed yield

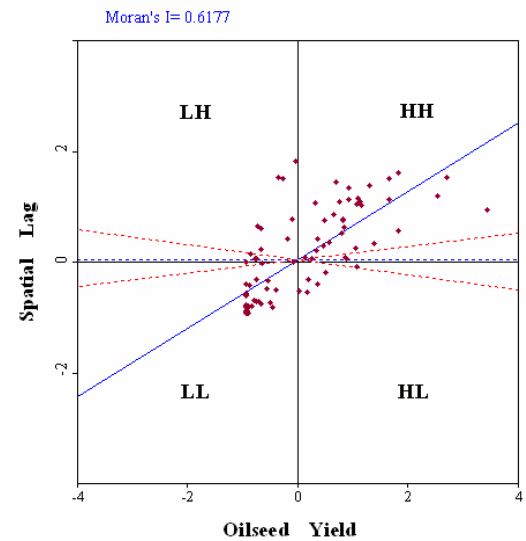

Fig. 4. Moran's I scatter plot of oilseed yield on country level

As shown in the study, there are important differences for time series, shocks to stationary time series are necessarily temporary, the mean or variance of a non-stationary series is time-dependent. To aid in the identification of a non-stationary series, we implemented the test analysis of unit root. In the paper, the unit root was carried out for the original data and the 1-order differential series data, then oilseed yield was verified as an integrated process, some typical parameters were selected during data transformation to eliminate the non-stationary characteristics, the computerization value of ADF test statistics is -1.872492 , is more than $1 \%$ (The critical value should be -3.670170 ), $5 \%$ (The critical value should be -2.963972 ), $10 \%$ (The critical value should be -2.621007$)$, which indicated the original data series was non-stationary. However, after 1-order differential transformation, the value of ADF test statistics is -7.031336 , is less than $1 \%$ (The critical value should be -3.679322 ), $5 \%$ (The critical value should be -2.967767$), 10 \%$ (The critical value should be -2.622989), which showed the time series data is in the stationary status. We also obtained the satisfactory stability after data transformation, or the characteristics roots of the polynomial must lie outside of the unit circle, the not oscillation series data was tested at the level of 0.01 , unit root test was appropriate statistics, so the total oilseed yield was the integrated sequence process.

\subsection{The Temporal Prognosis on ARIMA}

Based on the above the content of oilseed yield after 1-order differential for the temporal prognosis model of time series data combining with the lag orders, we obtained the appropriate identification $\operatorname{ARIMA}(2,1,2)$ model, for oilseed yield in Shandong province. Time series data in the study is regarded as the white noise characteristics after 1-order differential transformation, the estimated results by the model, with none of root is greater than 1, and conjugation root in the unit circle, so the smoothing process is within the parameters of the model, and through a significant test in the $95 \%$ confidence level and the relevant parameters of model were stationary, the 
estimation and prediction was carried out for oilseed yield, the results are as following, $\mathrm{D}($ oilseed yield,1) $=6.822+0.480 \mathrm{AR}(2)-0.971 \mathrm{MA}(2)$. Goodness of fit statistics was used to measure the advantages and disadvantages of model fitting. Generally, the key index was selected to satisfy the model accuracy, such as Akaike`s Information Criterion(AIC), DW, SC. Smaller the AIC value of model is, Better the result will be, in ARIMA model $, \mathrm{DW}=2.734, \mathrm{AIC}=10.423, \mathrm{SC}=10.56$, these values confirmed that the parameters of ARIMA model has goodness-of-fit [20,21].

\section{Spatial Agglomeration Mode of Oilseed Yield}

In our case, all Moran's I coefficients are significant in confidence level of the study. During the computerization, the spatial weight matrix is the fundamental tool used to represent the connectivity among country units, the weight matrix $w(k)$ which we use in this study is based on the $k$-nearest neighbors function as rules, the general form of the k-nearest neighbors weight matrix $w(k)$ is defined as follows:

$$
\begin{gathered}
w_{i j}(k)=0 \text { if } i=j \\
w_{i j}(k)=1 \text { if } d_{i j} \leq d_{i}(k) \text { and } d w_{i j}(k)=w_{i j}(k) / \sum_{j} w_{i j}(k) \\
w_{i j}(k)=0 \text { if } d_{i j}>d_{i}(k)
\end{gathered}
$$

Where $d_{i}(k)$ is a critical cut-off distance defined for each country unit $i$. More precisely, $d_{i}(k)$ is the $k^{\text {th }}$ order smallest distance between country units $i$ and $j$ such that each unit $i$ has exactly $k$ neighbors. Establishing the spatial weight file using a $k$-nearest neighbors contiguity matrix weights, which each observation has exactly the same number $(k)$ of neighbors. Alternatively, check the weights properties and the robustness of spatial country units [22].

It is vital to understand the regional effects for the spatial pattern of oilseed yield between country units, from the results of Moran's I coefficient analysis, spatial aggregating effect indicated the association of oilseed yield existed in adjacent to country units and the correlation relation was verified between observation vector and spatial lag vector based on Moran's I scatter plot. In addition to the inference computation, which is started by 999 -time Monte Carlo simulations, also note that the most significant p-level depends directly on the number of permutation, this will be $\mathrm{p}=0.01$, as shown in Figure 4., the value listed at the top of the graph $(0.6177)$ is the Moran's I statistics, which is regarded as the slope of the regression line, two dashed lines will appear in the plot, how the actual Moran scatter plot slope is well outside the range corresponding to the randomly permuted data. The autocorrelation results which indicated the spatial association of the oilseed yield was positively spatial autocorrelation (Moran's I value $=0.6177$, its theoretical mean value $=-0.0111$, the 
mean and standard deviation of the empirical distribution, these values are -0.0179 and 0.0745 , respectively).

\section{Results and Analysis}

As illustrated in Figure 4, the Moran scatter plot is a useful visualization tool for assessing distribution and spatial clustering of oilseed yield. In terms of the results of oilseed yield, Moran scatter plot characters of oilseed yield of 87 country units in 2007 showed the following concrete characters. The Moran's I coefficient of 14 country units was noted Quadrant I ( $\mathrm{HH}$, on the top right corner), which displays the country units with a high oilseed yield (above the average) surrounded by country units with high oilseed yield (above the average), as the following country units: Haiyang country, Qixia country, Pingyi country, Juxian country, Yinan country, Wulian country, Zhucheng city, Junan country, Gaomi city, Jimo city, Laiyang, Laixi city, Pingdu city, Laizhou city etc, which indicated the high oilseed yield value is in 14 country units and neighbouring country units, and the spatial difference is small between them. The Moran's I coefficient of 22 country units was noted Quadrant III(LL, on the bottom left), which presented the country units with low oilseed yield surrounded by country units with low oilseed yield. Spatial association of the LL type is observed in the following country units, Gaotang country, Xiajin country, Huimin country, Ningjin country, Pingyuan country, Shanghe country, Jiyang country, Qihe country, Yucheng city, Linyi city, Lingxian country, Wudi country, Yangxin country, Laoling city, Guangrao country, Zouping city, Huantai country, Qingyun country, Zhanhua country, Gaoqing country, Boxing country, Linjin country, etc. Quadrant IV (HL, on the bottom right) shows the country units with high value surrounded by country units with low values, the clustering mode of HL type country units involved in the following 13 country units Changdao country, Feixian country, Wulian country, Yinan country, Yishui country, Mengyin country, Pingyi country, Longkou city, Yiyuan country, Linqu country, Xintai city, Penglai city and Wengdeng city etc.. The Moran's I coefficient of 4 country units was noted Quadrant II (LH, on the top left corner), Tancheng country, Jiaozhou city, Changyi country and Mengyin country, which showed the country units with low value surrounded by country units with high values, the evident difference existed in them.

Moran's I quickly indicates not only the existence of spatial autocorrelation (positive or negative) but also the degree of spatial autocorrelation of oilseed yield showed spatial pattern of oilseed yield was not random on country level, and the higher oilseed yield on country level adjacent to the country level, the stronger spatial convergence of oilseed yield, the detailed spatial autocorrelation results of oilseed yield are the following: the Moran's I coefficient of 14 country units was noted $\mathrm{HH}$, which displays the country units with a high oilseed yield (above the average) surrounded by country units with high oilseed yield (above the average), and the spatial difference is low between the neighboring country units. The Moran's I coefficient of 22 country units was noted LL, which presented the country units with low oilseed yield surrounded by country units with low oilseed yield. 13 country units were the autocorrelation of HL type and the Moran's I coefficient of 4 country units was noted LH. In general, the more cluster trend was presented by the spatial autocorrelation 
characteristics analysis in 2007, the total difference of the oilseed yield presented the reasonable $\mathrm{HH}$ and LL tendency and the evident spatial convergence in the case study.

The temporal estimation and prediction of oilseed based on time series data was carried out for oilseed yield in Shandong province, the results are as following, $\mathrm{D}($ oilseed yield,1) $=6.822+0.480 \mathrm{AR}(2)-0.971 \mathrm{MA}(2)$. Goodness of fit statistics was used to measure the advantages and disadvantages of model fitting. Generally, the key index was selected to satisfy the model accuracy, such as Akaike`s Information Criterion(AIC), DW, SC. Smaller the AIC value of model is, Better the result will be, in the study, $\mathrm{DW}=2.734, \mathrm{AIC}=10.423, \mathrm{SC}=10.56$, these values confirmed the parameters of the goodness-of-fit model. The first-order univariate characteristics of oilseed yield was embodied with the help of time series data and the stationary process was validated by differential data, after first order differential, series data trend was eliminated and the relevant information was sufficiently extracted, series data was noted as white noise series. Finally $\operatorname{ARIMA}(2,1,2)$ was able to predict the growth tendency of oilseed yield, the oilseed yield changes as a time function from 1978 to 2008 was calculated by model, the results indicated that the oilseed yield growth kept in touch with the last oilseed yield, closely relationship was existed in the lag value of the first order and random interference item of oilseed yield in study country units, the average relative error between observation value and prediction value is $2.12 \%$ only using statistical oilseed yield data over 1978-2008, the better reliability after computation.

\section{Conclusions and Discussion}

In the paper, we analyzed the spatial contiguity characteristics with the support of Moran's I autocorrelation computation of oilseed yield on country level involving in 87 country units, and also considered modeling the long-run behavior of the total oilseed yield time series data on basis of $\operatorname{ARIMA}(2,1,2)$ model, and preliminary analyzed the temporal and spatial characteristics of oilseed yield in Shandong province.

For spatial pattern of oilseed yield, it is clear that spatial autocorrelation of oilseed yield of country units that fall into the quadrants HH and LL, which represented clustering of high and low value respectively, 14 country units (quadrant $\mathrm{HH}$ ), 22 country units (quadrant LL) of oilseed yield presented high spatial clustering, 4 country units (quadrant $\mathrm{LH}$ ) and 13 country units(quadrant HL), HL and LH represent the negative association with their neighbors and can be considered as spatial outliers, there is no evident clustering characteristics of the oilseed yield for other 34 county units, so we should sum up the more suitable regional development countermeasure on the spatial autocorrelation effects of Moran's I statistics value for upgrading economic crops industry structure.

In addition, the other characteristics of the paper indicated that a great amount of historical data of oilseed yield was analyzed by means of $\operatorname{ARIMA}(2,1,2)$ model, and established the model and studied the trend of the changes of oilseed yield. The advantage of the $\operatorname{ARIMA}(2,1,2)$ model we created in this paper is simple, practical, and will have a wide range of application, which is used as the exponential smoothing and seasonal index to predict the oilseed yield. We have also estimated the equation for a subset of our data, so that we may compare prediction value based upon this model with 
the actual data over 1978-2008. This proved that our model is effective and feasible to predict the future yield. In a word, the spatial and temporal prediction of oilseed yield is developed comparison study and complement to each other, resulting in a good agreement with the experimental data. In addition, the study is to provide a better understanding of temporal and spatial patterns of oilseed yield in Shandong province.

\section{References}

[1] Wan, S., et al.: The high Quality of synergism Cultivation Theory and Technology of Peanut. China Agricultural Scientific Press, Beijing (2009)

[2] Fischer, M.M., Stirböck, C.: Pan-European regional income growth and club-convergence. Ann. Reg. Sci. 40, 693-721 (2006)

[3] Ertur, C., Koch, W.: Regional disparities in the European Union and the enlargement process: an exploratory spatial data analysis. Ann. Reg. Sci. 40, 723-765 (2006)

[4] Wang, J.: Spital Analysis. Science Press, Beijing (2006) (in Chinese

[5] Wu, Y., Li, J.: A spatial econometric analysis of industrial total factor productivity in China's provincial regions. Scientia Geographica Sinica 26(4), 385-391 (2006) (in Chinese)

[6] Li, Y., Lv, A., Bian, X.: Regional Changes of Per Capita Grain Possession and Its Driving Mechanisms in Jiangsu Province. Resources Science 30(3), 423-431 (2008) (in Chinese)

[7] Chen, G., XuGuowei, X., Chen, W.H.: The Corn Output in A Time Series Prediction Model. In: Li, D., Yang, S.X. (eds.) Computer and computing technologies in agriculture, pp. 141-147. Tsi Press Series, TX USA (2010)

[8] Brockwell, P.J., Davis, R.A.: Time Series:Theory and Methods, 2nd edn. Springer, Heidelberg (2001)

[9] Gao, T.: Eviews Application and Cases of Quantity Economic Analysis and Model. Qinghua University Press, Beijing (2005)

[10] Zhang, S., Qi, L.: A Concise Guide of Time series analysis, pp. 1-80. Tsinghua University Press, Beijing (2003)

[11] Brockwell, P.J., Davis, R.A.: Introduction to Time Series and Forecasting, 8th edn. Springer, New York Sections 3.3 and 8.3. (1996)

[12] Zhang, X.: A Guide to Using Eviews. China Machine Press, Beijing (2007)

[13] $\mathrm{Wu}, \mathrm{Y} ., \mathrm{Li}, \mathrm{J} .:$ A spatial econometric analysis of industrial total factor productivity in China's provincial regions. Scientia Geographica Sinica 26(4), 385-391 (2006)

[14] Ma, R., Pu, Y., Ma, X.: Developing the Spatial Association Pattern based on GIS. Science Press, Beijing (2007)

[15] Li, Y., Lv, A., Bian, X.: Regional Changes of Per Capita Grain Possession and Its Driving Mechanisms in Jiangsu Province. Resources Science 30(3), 423-431 (2008)

[16] Haining, R.P.: Spatial data analysis: theory and practice. Cambridge University Press, Cambridge (2003)

[17] Brasier, K.J.: Spatial Analysis of Changes in the Number of Farms During the Farm Crisis. Rural Sociology 70(4), 540-560 (2005)

[18] Anselin, L., Florax, R.J.G.M., Rey, S.J.: Advances in spatial econometics: methodology, tools and applications. Springer Overlag, Berlin (2004)

[19] Eviews 6.0 User's Guide,Quantiative Micro Software (2009) 
[20] Pucheta, J., Patio, D., Kuchen, B.: A statistically dependent approach for the monthly rainfall forcast from one point observations. In: Li, D., Yang, S.X. (eds.) Computer and computing technologies in agriculture, pp. 141-147. Tsi Press Series, TX

[21] Ertur, C., Koch, W.: Regional disparities in the European Union and the enlargement process: an exploratory spatial data analysis. Ann. Reg. Sci. 40, 723-765 (2006)

[22] Russo, G., Reggiani, A., Nijkamp, P.: Spatial activity and labour market patterns: A connectivity analysis of commuting flows in Germany. Ann. Reg. Sci. 41, 789-811 (2007) 